\title{
A practical synthesis and the pharmacological potency of gem-diphosphono substituted pyrimidines for treatment of bone resorption
}

\author{
Azza A. Kamel* and Maha D. Khidre \\ Pesticide Chemistry Department, National Research Centre, Dokki, D - 12622, Cairo, Egypt, \\ Fax: 20237601877 \\ E-mail: azza_kamel@yahoo.com
}

\begin{abstract}
A series of substituted arylidenes was allowed to react with the Wittig-Horner reagent, tetraethylmethylene-1,1-bisphosphonate to give the corresponding $N$-containing bisphosphonates (BPs). Acid hydrolysis of one of the BPs gave the corresponding bisphosphonic acid. The in vivo antiresorptive activities of the products in the rat adjuvant model are discussed in terms of structureactivity relationships (SAR).
\end{abstract}

Keywords: Wittig-Horner carbanions, heteroarylmethylene 1,1-bisphosphonates, nitrogencontaining bisphosphonates, bone resorption

\section{Introduction}

Through the past three decades, the discovery and development of bisphosphonates (BPs), and their related bisphosphonic acids as a class of compounds for treatment of bone diseases has been a fascinating saga in medicinal chemistry. ${ }^{1-3}$ The clinical pharmacology of BPs is characterized by low intestinal absorption, but they inhibit bone resorption by being highly selectively taken up and adsorbed to mineral surfaces in bone, where they interfere as osteoclasts. It is likely that BPs are internalized by osteoclasts and interfere with specific biochemical processes and thereby induced programmed cell death or apoptosis. ${ }^{4,5}$

Recent studies show that BPs can be classified into at least two groups with different modes of action: (a) BPs that most closely resemble pyrophosphates (such as clodronate and etidronate, see Fig.1) can be metabolically incorporated into non-hydrolysable analogs of ATP that may inhibit ATP-dependant intercellular enzymes. (b) The more potent, $N$-containing bisphosphonates (such as pamidronate and risedronate, see Fig.2) are not metabolized in this way but can act on liver enzymes function, which explains the loss of osteoclast activity and induction of apoptosis. 
<smiles>O=P([O-])([O-])C(Cl)(Cl)P(=O)([O-])[O-]</smiles>

Clodronate<smiles>CC(O)(P(=O)([O-])[O-])P(=O)([O-])[O-]</smiles>

Etidronate

\section{Figure 1}

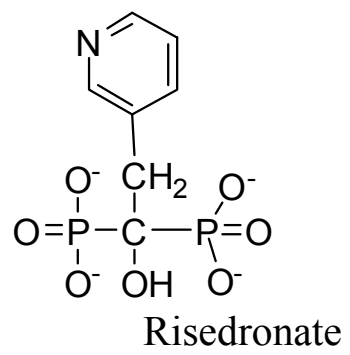<smiles>NCCCC(O)(P(=O)([O-])[O-])P(=O)([O-])[O-]</smiles>

Pamidronate

\section{Figure 2}

A series of articles ${ }^{6,7}$ from our laboratory has reported simple and efficient procedures for the synthesis of several examples of $N$-heterocycle- substituted-methylene-1,1-bisphosphonic acids $\mathbf{3}$ derived from the reaction of the parent olefin containing different heterocyclic species $\mathbf{2}$ with tetraethyl methylene-1,1-bisphosphonate, 1, followed by acid hydrolysis, as shown in Scheme 1.<smiles>CCO[PH](=O)C[PH](=O)OCC</smiles>

1

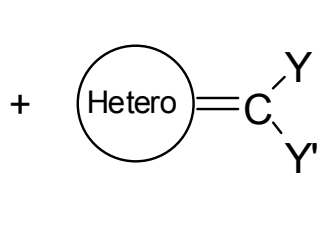

2<smiles></smiles>

3

\section{Scheme 1}

In continuation of our research program ${ }^{6-9}$ on synthesis of a variety of bisphosphonates and their related bisphosphonic acids, it was of interest to introduce another series of these compounds for investigating the structure-activity relationships (SAR). In this work, we have attempted to utilize the high bone-joint- specificity of nitrogen- containing BPs with other 
substituents of potential anti-catabolic pharmacology for testing a class of compounds for the treatment of human bone diseases.

\section{Results and Discussion}

The synthesis of the required nitrogen containing bisphosphonates (BPs) was achieved via the Michael addition of a small excess over a molar amount of the Wittig-Horner reagent $\mathbf{1}$ to acids 4a-f or their N-methyl analogues $\mathbf{4 g - j}$. The reaction was carried out under reflux, with basic catalysis using DMSO as solvent and $\mathrm{LiOH}(0.5 M)$ as a base. After the reaction was completed (TLC) and the product mixture acid- quenched, the crude BP product was separated by solvent extraction and purified by column chromatography to give tetraethyl 2-aryl-2-(2,4,6-trioxohexahydropyrimidin-5-yl)ethyl-1,1-bisphosphonate $\mathbf{6 a - j}$ as a major product $(\approx 65 \%)$ together with a very low yield of tetraethyl 2-arylidene-bisphosphonate 8a-f $(\leq 7 \%)$. Acid hydrolysis (conc. $\mathrm{HCl}$ ) of the resulting BP product $\mathbf{6} \mathbf{h}$ - taken as an example - afforded the corresponding BP-acid 7 ( $\approx 90 \%$, based on $6 \mathbf{h})$ (Scheme 2$)$.<smiles>[Y]c1ccc(C=C2C(=O)NC(=O)NC2=O)cc1</smiles>

$4 a-j$

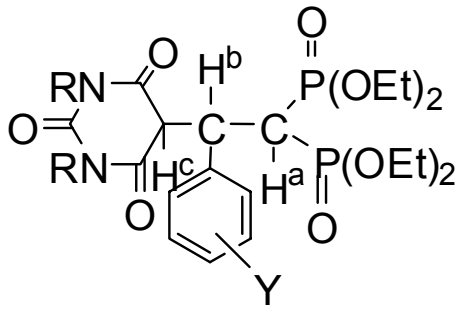

6a-j ( 65\%)

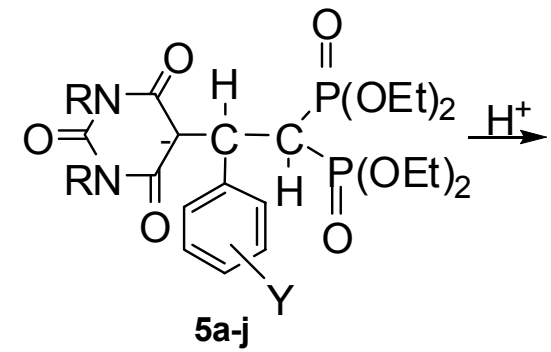

5a-j<smiles>[Y]c1ccc(C=C(P(=O)=O)[PH](=O)OCC)cc1</smiles>

8a-f $(\leq 7 \%)$

, Y as in 4

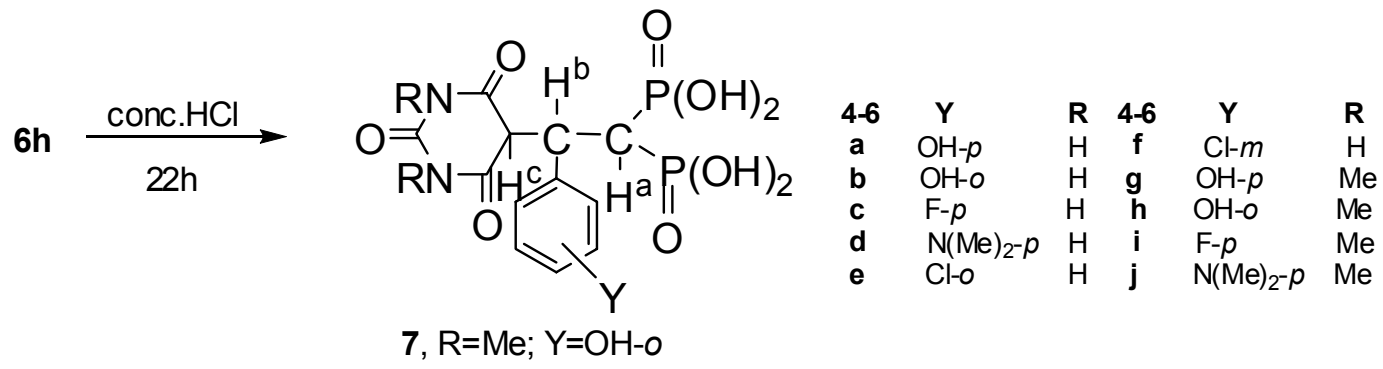

Scheme 2 
The structures of compounds 6a-j were verified by NMR analysis. For instance, the ${ }^{1} \mathrm{H}-\mathrm{NMR}$ (400 MHz, DMSO) spectrum of $\mathbf{6 a}$ revealed three types of methine protons at $\delta=2.99$ $\left(\mathrm{dd}, J_{H H}=9 \mathrm{~Hz},{ }^{2} J_{H P}=13.7 \mathrm{~Hz}, 1 \mathrm{H}, H^{a} \mathrm{C}\right), 3.87\left(\mathrm{ddd}, J_{H H}=9,11 \mathrm{~Hz}, 1 \mathrm{H}, \mathrm{H}^{\mathrm{b}} \mathrm{C}\right), 4.89(\mathrm{dd}$, $\left.J_{H H}=11 \mathrm{~Hz}, 1 \mathrm{H}, \mathrm{H}^{\mathrm{c}} \mathrm{C}\right) \mathrm{ppm}$. The large coupling constant $\left(J_{H H}\right)$ of $\mathrm{H}^{\mathrm{b}}$ with $\mathrm{H}_{\mathrm{a}}$ as well as with $\mathrm{H}^{\mathrm{c}}$ (see Table 3) indicates ${ }^{10}$ that $\mathrm{H}^{\mathrm{b}}$ is trans to $\mathrm{H}_{\mathrm{a}}$ and $\mathrm{H}^{\mathrm{c}}$. On the other hand, IR and NMR analysis established the structure of ethenylidenebisphosphonates $\mathbf{8}$. The IR ( $\mathrm{KBr}$ ) spectrum of $\mathbf{8 a}$ (as an example) showed the absence of stretching bands in the range $1700-1650 \mathrm{~cm}^{-1}$ corresponding to the three $\mathrm{C}=\mathrm{O}$ groups of the starting arylidene 4a. Furthermore, in the ${ }^{1} \mathrm{H}$ NMR spectrum, only one methine proton could be indicated at $8.1\left(\mathrm{dd}, 1 \mathrm{H}, H \mathrm{C}=\mathrm{CP}_{2}\right) \mathrm{ppm}$. The ${ }^{13} \mathrm{C} \mathrm{NMR}$ as well as MS spectra were in agreement with the proposed structure.

Obviously, the bisphosphosphonate products $\mathbf{6 a}-\mathbf{j}$ were formed according to a process derived from the Michael addition ${ }^{11 a}$ of arylidine 4 to tetraethyl methylenebisphosphonate (1) to give anion $\mathbf{5}$. When $\mathbf{5}$ is quenched by an acid at low temperature, the target bisphosphonate product 6 is obtained. On the other hand, the long time of heating ( $\approx 2$ days) and the presence of a base might be responsible for the formation of the isolated by-product ethenylidenebisphosphonates $\mathbf{8}$. This could be attributed ${ }^{11 \mathrm{~b}}$ to the intramolecular proton transfer in anion $\mathbf{5}$ to form the more stable form $\mathbf{5 A}$; when anion $\mathbf{5 A}$ is heated, it splits off to form BP $\mathbf{8}$ together with a trace amount of acid 9a or its dimethyl analog 9b (mp, TLC, MS and IR spectrum for $9 \mathbf{a}$ or $\mathbf{9 b}$ were found to be identical with an authentic sample). The low yield of product $8(\leq 7 \%)$ indicates that the reaction seems to be a reversible process. Although Hutchinson et al. ${ }^{12}$ reported the inertness of ethenylidenebisphosphonate toward carbanions, Stutz achieved the Michael condensation product using some carbanions in high yields. ${ }^{13}$ These two contradictory results may best be rationalized by an equilibrium process similar to that shown in Scheme 3. Different results may be obtained with various carbanions or under different experimental conditions.

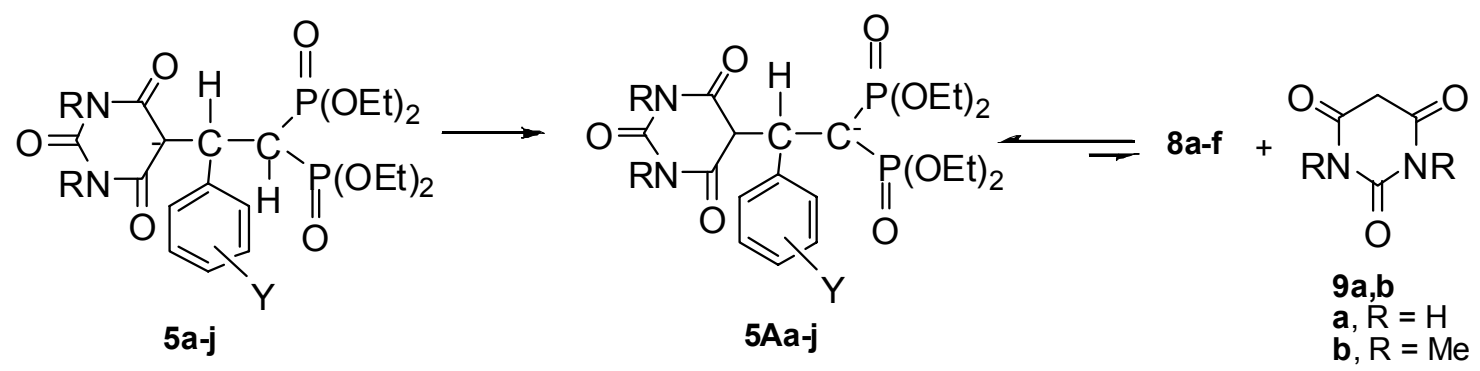

\section{Scheme 3}

In order to verify our suggestion, we added $p$-nitrobenzaldehyde to the reaction solution $(\mathbf{1}+\mathbf{4 a})$ that underwent Wittig-Horner reaction with anion 5A. As a result, the equilibrium between $\mathbf{5 A}$ and $\mathbf{8}$ was shifted to the left side completely to give the expected Wittig-Horner monophosphonate $\mathbf{1 0}$ as a sole product (Scheme 4). This is a strong evidence supporting our 
postulation. On the other hand, if we could drive the equilibrium completely towards the formation of compound $\mathbf{8}$, it would be of synthetic importance since only a few methods are available for the preparation of ethenylidenebisphosphonates $8{ }^{14}$ However our trial on just raising the reaction temperature failed to give the expected result.

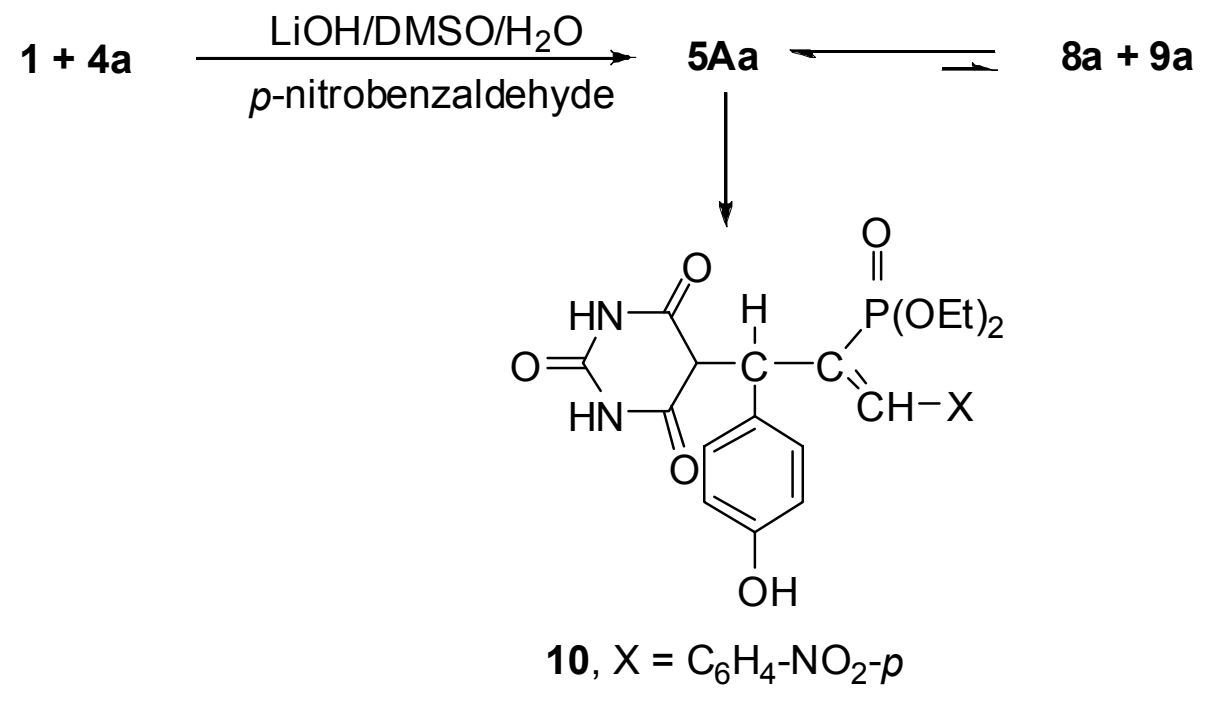

\section{Scheme 4}

The structures suggested for all the new compounds are in good agreement with their analytical and spectroscopic data (Tables 1-3).

Table 1. Physical properties and elemental analyses for compounds: 6a-j, 7, 8a-f and 10

\begin{tabular}{|c|c|c|c|c|c|c|c|c|c|}
\hline \multirow{2}{*}{$\begin{array}{l}\text { Product, } \\
\text { color }\end{array}$} & \multirow{2}{*}{$\begin{array}{c}\text { Yiel } \\
\text { d } \\
(\%)\end{array}$} & \multirow{2}{*}{$\begin{array}{c}\mathrm{mp} \\
\left({ }^{\circ} \mathrm{C}\right), \\
\text { solvent }\end{array}$} & \multirow{2}{*}{$\begin{array}{l}\text { Mol. Formula } \\
\text { (Mol. wt.) }\end{array}$} & \multicolumn{5}{|c|}{ Anal. Found / Calcd. \% } & \multirow[b]{2}{*}{$\mathrm{P}$} \\
\hline & & & & $\mathrm{C}$ & $\mathrm{H}$ & $\mathrm{Cl}$ & $\mathrm{F}$ & $\mathrm{N}$ & \\
\hline \multirow{4}{*}{$\begin{array}{c}\mathbf{6 a}, \\
\text { yellow } \\
\mathbf{6 b}, \\
\text { yellow }\end{array}$} & \multirow[t]{2}{*}{65} & $>300$ & \multirow{2}{*}{$\begin{array}{l}\mathrm{C}_{20} \mathrm{H}_{30} \mathrm{~N}_{2} \mathrm{O}_{10} \mathrm{P}_{2} \\
(520.41)\end{array}$} & 46.23 & 5.88 & - & - & 5.25 & 11.79 \\
\hline & & $\mathrm{MeCN}$ & & 46.16 & 5.81 & - & - & 5.38 & 11.90 \\
\hline & \multirow[t]{2}{*}{55} & $>300$ & \multirow{2}{*}{$\begin{array}{l}\mathrm{C}_{20} \mathrm{H}_{30} \mathrm{~N}_{2} \mathrm{O}_{10} \mathrm{P}_{2} \\
(520.41)\end{array}$} & 46.19 & 5.83 & - & - & 5.30 & 11.85 \\
\hline & & $\mathrm{MeCN}$ & & 46.16 & 5.81 & - & - & 5.38 & 11.90 \\
\hline \multirow[b]{2}{*}{$\begin{array}{c}\mathbf{6 c}, \\
\text { yellow }\end{array}$} & \multirow{2}{*}{62} & $205-$ & \multirow{2}{*}{$\begin{array}{l}\mathrm{C}_{20} \mathrm{H}_{29} \mathrm{FN}_{2} \mathrm{O}_{9} \mathrm{P}_{2} \\
(522.40)\end{array}$} & 45.86 & 5.64 & - & 3.48 & 5.29 & 11.80 \\
\hline & & $\begin{array}{l}207 \\
\mathrm{EtOH}\end{array}$ & & 45.98 & 5.60 & - & 3.64 & 5.36 & 11.86 \\
\hline \multirow{2}{*}{$\begin{array}{c}\mathbf{6 d}, \\
\text { orange }\end{array}$} & \multirow{2}{*}{70} & $260-$ & \multirow{2}{*}{$\begin{array}{l}\mathrm{C}_{22} \mathrm{H}_{35} \mathrm{~N}_{3} \mathrm{O}_{9} \mathrm{P}_{2} \\
(547.48)\end{array}$} & 48.35 & 6.51 & - & - & 7.57 & 11.19 \\
\hline & & $\begin{array}{l}262, \\
\mathrm{EtOH}\end{array}$ & & 48.26 & 6.44 & - & - & 7.68 & 11.32 \\
\hline \multirow{2}{*}{$\begin{array}{l}\mathbf{6 e}, \text { pale } \\
\text { yellow }\end{array}$} & \multirow{2}{*}{50} & $224-$ & \multirow{2}{*}{$\begin{array}{l}\mathrm{C}_{20} \mathrm{H}_{29} \mathrm{ClN}_{2} \mathrm{O}_{9} \mathrm{P}_{2} \\
(538.85)\end{array}$} & 44.66 & 5.39 & 6.47 & - & 5.12 & 11.38 \\
\hline & & $\begin{array}{l}226 \\
\text { EtOAc }\end{array}$ & & 44.58 & 5.42 & 6.58 & - & 5.20 & 11.50 \\
\hline
\end{tabular}




\begin{tabular}{|c|c|c|c|c|c|c|c|c|c|}
\hline $\begin{array}{c}\mathbf{6 f}, \\
\text { yellow }\end{array}$ & 55 & $\begin{array}{l}232- \\
234 \\
\text { EtOAc }\end{array}$ & $\begin{array}{l}\mathrm{C}_{20} \mathrm{H}_{29} \mathrm{ClN}_{2} \mathrm{O}_{9} \mathrm{P}_{2} \\
(538.85)\end{array}$ & $\begin{array}{l}44.68 \\
44.58\end{array}$ & $\begin{array}{l}5.32 \\
5.42\end{array}$ & $\begin{array}{l}6.44 \\
6.58\end{array}$ & $\begin{array}{l}- \\
-\end{array}$ & $\begin{array}{l}5.09 \\
5.20\end{array}$ & $\begin{array}{l}11.33 \\
11.50\end{array}$ \\
\hline $\begin{array}{c}\mathbf{6 g}, \\
\text { yellow }\end{array}$ & 48 & $\begin{array}{l}261- \\
263, \\
\text { EtOH }\end{array}$ & $\begin{array}{l}\mathrm{C}_{22} \mathrm{H}_{34} \mathrm{~N}_{2} \mathrm{O}_{10} \mathrm{P}_{2} \\
(548.46)\end{array}$ & $\begin{array}{l}48.23 \\
48.18\end{array}$ & $\begin{array}{l}6.31 \\
6.25\end{array}$ & $\begin{array}{l}- \\
-\end{array}$ & $\begin{array}{l}- \\
-\end{array}$ & $\begin{array}{l}5.09 \\
5.11\end{array}$ & $\begin{array}{l}11.18 \\
11.29\end{array}$ \\
\hline $\begin{array}{c}\mathbf{6 h}, \\
\text { orange }\end{array}$ & 44 & $\begin{array}{l}275- \\
277, \\
\text { EtOH }\end{array}$ & $\begin{array}{l}\mathrm{C}_{22} \mathrm{H}_{34} \mathrm{~N}_{2} \mathrm{O}_{10} \mathrm{P}_{2} \\
(548.46)\end{array}$ & $\begin{array}{l}48.29 \\
48.18\end{array}$ & $\begin{array}{l}6.35 \\
6.25\end{array}$ & $\begin{array}{l}- \\
-\end{array}$ & $\begin{array}{l}- \\
-\end{array}$ & $\begin{array}{l}5.05 \\
5.11\end{array}$ & $\begin{array}{l}11.14 \\
11.29\end{array}$ \\
\hline $\begin{array}{l}\text { 6i, straw } \\
\text { yellow }\end{array}$ & 65 & $\begin{array}{l}125- \\
126, \\
\mathrm{CHCl}_{3}\end{array}$ & $\begin{array}{l}\mathrm{C}_{22} \mathrm{H}_{33} \mathrm{FN}_{2} \mathrm{O}_{9} \mathrm{P}_{2} \\
(550.45)\end{array}$ & $\begin{array}{l}48.11 \\
48.00\end{array}$ & $\begin{array}{l}6.15 \\
6.04\end{array}$ & $\begin{array}{l}- \\
-\end{array}$ & $\begin{array}{l}3.32 \\
3.45\end{array}$ & $\begin{array}{l}5.15 \\
5.09\end{array}$ & $\begin{array}{l}11.18 \\
11.25\end{array}$ \\
\hline $\begin{array}{l}\mathbf{6 j} \text {, } \\
\text { reddish/ } \\
\text { brown }\end{array}$ & 72 & $\begin{array}{l}201- \\
203, \\
\text { AcOEt }\end{array}$ & $\begin{array}{l}\mathrm{C}_{24} \mathrm{H}_{39} \mathrm{~N}_{3} \mathrm{O}_{9} \mathrm{P}_{2} \\
(575.53)\end{array}$ & $\begin{array}{l}50.21 \\
50.09\end{array}$ & $\begin{array}{l}6.79 \\
6.83\end{array}$ & $\begin{array}{l}- \\
-\end{array}$ & $\begin{array}{l}- \\
-\end{array}$ & $\begin{array}{l}7.18 \\
7.30\end{array}$ & $\begin{array}{l}10.64 \\
10.76\end{array}$ \\
\hline $\begin{array}{c}\text { 7, } \\
\text { colorless }\end{array}$ & $80^{\mathrm{a}}$ & $\begin{array}{l}>300 \\
\mathrm{MeOH}\end{array}$ & $\begin{array}{l}\mathrm{C}_{14} \mathrm{H}_{18} \mathrm{~N}_{2} \mathrm{O}_{10} \mathrm{P}_{2} \\
(436.25)\end{array}$ & $\begin{array}{l}38.42 \\
38.54\end{array}$ & $\begin{array}{l}4.22 \\
4.16\end{array}$ & $\begin{array}{l}- \\
-\end{array}$ & $\begin{array}{l}- \\
-\end{array}$ & $\begin{array}{l}6.47 \\
6.42\end{array}$ & $\begin{array}{l}14.08 \\
14.20\end{array}$ \\
\hline $\begin{array}{c}\mathbf{8 a}, \\
\text { colorless }\end{array}$ & 7 & $\begin{array}{l}>300 \\
\text { EtOH }\end{array}$ & $\begin{array}{l}\mathrm{C}_{16} \mathrm{H}_{26} \mathrm{O}_{7} \mathrm{P}_{2} \\
(392.32)\end{array}$ & $\begin{array}{l}49.11 \\
48.98\end{array}$ & $\begin{array}{l}6.77 \\
6.68\end{array}$ & - & - & $\begin{array}{l}- \\
-\end{array}$ & $\begin{array}{l}15.68 \\
15.79\end{array}$ \\
\hline $\begin{array}{l}\mathbf{8 b}, \text { pale } \\
\text { yellow }\end{array}$ & 8 & $\begin{array}{l}>300 \\
\text { EtOH }\end{array}$ & $\begin{array}{l}\mathrm{C}_{16} \mathrm{H}_{26} \mathrm{O}_{7} \mathrm{P}_{2} \\
(392.32)\end{array}$ & $\begin{array}{l}49.05 \\
48.98\end{array}$ & $\begin{array}{l}6.72 \\
6.68\end{array}$ & $\begin{array}{l}- \\
-\end{array}$ & $\begin{array}{l}- \\
-\end{array}$ & $\begin{array}{l}- \\
-\end{array}$ & $\begin{array}{l}15.65 \\
15.79\end{array}$ \\
\hline $\begin{array}{l}\mathbf{8 c} \text {, pale } \\
\text { yellow }\end{array}$ & $\leq 5$ & $\begin{array}{l}139- \\
140 \\
c- \\
\text { hexane }\end{array}$ & $\begin{array}{l}\mathrm{C}_{16} \mathrm{H}_{25} \mathrm{FO}_{6} \mathrm{P}_{2} \\
(394.31)\end{array}$ & $\begin{array}{l}49.83 \\
48.74\end{array}$ & $\begin{array}{l}6.46 \\
6.39\end{array}$ & $\begin{array}{l}- \\
-\end{array}$ & $\begin{array}{l}- \\
-\end{array}$ & $\begin{array}{l}- \\
-\end{array}$ & $\begin{array}{l}15.59 \\
15.71\end{array}$ \\
\hline $\begin{array}{c}\mathbf{8 d}, \\
\text { yellow }\end{array}$ & $\leq 5$ & $\begin{array}{l}240- \\
242, \\
\mathrm{CHCl}_{3}\end{array}$ & $\begin{array}{l}\mathrm{C}_{18} \mathrm{H}_{31} \mathrm{NO}_{6} \mathrm{P}_{2} \\
(419.39)\end{array}$ & $\begin{array}{l}51.68 \\
51.55\end{array}$ & $\begin{array}{l}7.53 \\
7.45\end{array}$ & - & $\begin{array}{l}- \\
-\end{array}$ & $\begin{array}{l}3.21 \\
3.34\end{array}$ & $\begin{array}{l}14.59 \\
14.77\end{array}$ \\
\hline $\begin{array}{c}\mathbf{8 e}, \\
\text { colorless }\end{array}$ & 7 & $\begin{array}{l}162- \\
164, \\
\mathrm{CH}_{2} \mathrm{Cl}_{2}\end{array}$ & $\begin{array}{l}\mathrm{C}_{16} \mathrm{H}_{25} \mathrm{ClO}_{6} \mathrm{P}_{2} \\
(410.77)\end{array}$ & $\begin{array}{l}46.91 \\
46.78\end{array}$ & $\begin{array}{l}6.21 \\
6.13\end{array}$ & $\begin{array}{l}8.51 \\
8.63\end{array}$ & $\begin{array}{l}- \\
-\end{array}$ & - & $\begin{array}{l}14.89 \\
15.08\end{array}$ \\
\hline $\begin{array}{c}\mathbf{8 f}, \\
\text { colorless }\end{array}$ & 6 & $\begin{array}{l}178- \\
180 \\
\mathrm{CH}_{2} \mathrm{Cl}_{2}\end{array}$ & $\begin{array}{l}\mathrm{C}_{16} \mathrm{H}_{25} \mathrm{ClO}_{6} \mathrm{P}_{2} \\
(410.77)\end{array}$ & $\begin{array}{l}46.82 \\
46.78\end{array}$ & $\begin{array}{l}6.19 \\
6.13\end{array}$ & $\begin{array}{l}8.49 \\
8.63\end{array}$ & $\begin{array}{l}- \\
-\end{array}$ & $\begin{array}{l}- \\
-\end{array}$ & $\begin{array}{l}14.92 \\
15.08\end{array}$ \\
\hline $\begin{array}{c}\mathbf{1 0}, \\
\text { yellow }\end{array}$ & 60 & $\begin{array}{l}274- \\
275, \\
\text { EtOH }\end{array}$ & $\begin{array}{l}\mathrm{C}_{23} \mathrm{H}_{24} \mathrm{~N}_{3} \mathrm{O}_{9} \mathrm{P} \\
(517.425)\end{array}$ & $\begin{array}{l}53.51 \\
53.39\end{array}$ & $\begin{array}{l}4.77 \\
4.68\end{array}$ & - & $\begin{array}{l}- \\
-\end{array}$ & $\begin{array}{l}8.08 \\
8.12\end{array}$ & $\begin{array}{l}5.81 \\
5.99\end{array}$ \\
\hline
\end{tabular}

${ }^{a}$ Yield is based on the consumed corresponding bisphosphonate $\mathbf{6 h}$. 
Table 2. IR and mass spectra for compounds $6 \mathbf{a}-\mathbf{j}, \mathbf{7}, \mathbf{8 a - f}$ and $\mathbf{1 0}$

\begin{tabular}{|c|c|c|}
\hline $\begin{array}{c}\text { Comp. } \\
\text { no. }\end{array}$ & $\begin{array}{l}\text { MS: } m / z \\
\%[\text { ion }]\end{array}$ & $\operatorname{IR}(\mathrm{KBr}) v_{\max } / \mathrm{cm}^{-1}$ \\
\hline 6a & $\begin{array}{l}520(11) \\
{\left[\mathrm{M}^{+}\right]}\end{array}$ & $\begin{array}{l}3455-3358(\mathrm{OH} \text { and } \mathrm{NH}), 1706,1690,1665(3 \times \mathrm{C}=\mathrm{O}), 1264(\mathrm{P}=\mathrm{O}), 1144 \\
1083(\mathrm{P}-\mathrm{O}-\mathrm{C})\end{array}$ \\
\hline $\mathbf{6 b}$ & $\begin{array}{l}520(17) \\
{\left[\mathrm{M}^{+}\right]}\end{array}$ & $\begin{array}{l}3440-3365(\mathrm{OH} \text { and } \mathrm{NH}), 1708,1685,1664(3 \times \mathrm{C}=\mathrm{O}), 1262(\mathrm{P}=\mathrm{O}), 1150 \\
1080(\mathrm{P}-\mathrm{O}-\mathrm{C})\end{array}$ \\
\hline 6c & $\begin{array}{l}522(5) \\
{\left[\mathrm{M}^{+}\right]}\end{array}$ & $\begin{array}{l}3360_{\mathrm{w}}(\mathrm{NH}), 1710,1683,1662(3 \times \mathrm{C}=\mathrm{O}), 1618(\mathrm{C}=\mathrm{C}), 1263(\mathrm{P}=\mathrm{O}), 1148, \\
1082(\mathrm{P}-\mathrm{O}-\mathrm{C})\end{array}$ \\
\hline 6d & $\begin{array}{l}547(15) \\
{\left[\mathrm{M}^{+}\right]}\end{array}$ & $\begin{array}{l}3358_{\mathrm{w}}(\mathrm{NH}), 1708,1685,1664(3 \times \mathrm{C}=\mathrm{O}), 1637(\mathrm{C}=\mathrm{C}), 1266(\mathrm{P}=\mathrm{O}), 1155, \\
1085(\mathrm{P}-\mathrm{O}-\mathrm{C})\end{array}$ \\
\hline $6 e$ & $\begin{array}{l}538(7) \\
{\left[\mathrm{M}^{+}\right]}\end{array}$ & $\begin{array}{l}3364_{\mathrm{w}}(\mathrm{NH}), 1700,1690,1652(3 \times \mathrm{C}=\mathrm{O}), 1625(\mathrm{C}=\mathrm{C}), 1258(\mathrm{P}=\mathrm{O}), 1160 \text {, } \\
1083(\mathrm{P}-\mathrm{O}-\mathrm{C})\end{array}$ \\
\hline 6f & $\begin{array}{l}538(6) \\
{\left[\mathrm{M}^{+}\right]}\end{array}$ & $\begin{array}{l}3359_{\mathrm{w}}(\mathrm{NH}), 1720,1685,1660(3 \times \mathrm{C}=\mathrm{O}), 1618(\mathrm{C}=\mathrm{C}), 1263(\mathrm{P}=\mathrm{O}), 1158 \text {, } \\
1086(\mathrm{P}-\mathrm{O}-\mathrm{C})\end{array}$ \\
\hline $6 \mathbf{g}$ & $\begin{array}{l}548(21) \\
{\left[\mathrm{M}^{+}\right]}\end{array}$ & $\begin{array}{l}\text { 1760, 1690, } 1672(3 \times \mathrm{C}=\mathrm{O}), 1622(\mathrm{C}=\mathrm{C}), 1248(\mathrm{P}=\mathrm{O}), 1148, \\
1080(\mathrm{P}-\mathrm{O}-\mathrm{C})\end{array}$ \\
\hline $6 \mathbf{h}$ & $\begin{array}{l}548(24) \\
{\left[\mathrm{M}^{+}\right]}\end{array}$ & $\begin{array}{l}1725,1682,1665(3 \times \mathrm{C}=\mathrm{O}), 1618(\mathrm{C}=\mathrm{C}), 1253(\mathrm{P}=\mathrm{O}), 1145, \\
1075(\mathrm{P}-\mathrm{O}-\mathrm{C})\end{array}$ \\
\hline $6 \mathbf{i}$ & $\begin{array}{l}550(9) \\
{\left[\mathrm{M}^{+}\right]}\end{array}$ & $\begin{array}{l}1719,1683,1662(3 \times \mathrm{C}=\mathrm{O}), 1615(\mathrm{C}=\mathrm{C}), 1260(\mathrm{P}=\mathrm{O}), 1150, \\
1078 \quad(\mathrm{P}-\mathrm{O}-\mathrm{C})\end{array}$ \\
\hline $6 \mathbf{j}$ & $\begin{array}{l}575(19) \\
{\left[\mathrm{M}^{+}\right]}\end{array}$ & $1709,1678,1659(3 \times \mathrm{C}=\mathrm{O}), 1640(\mathrm{C}=\mathrm{C}), 1250(\mathrm{P}=\mathrm{O}), 1139,1080(\mathrm{P}-\mathrm{O}-\mathrm{C})$ \\
\hline 7 & $\begin{array}{l}436(18) \\
{\left[\mathrm{M}^{+}\right]}\end{array}$ & $3330-3220(\mathrm{P}-\mathrm{OH})$, and 1224-1235 $(\mathrm{P}=\mathrm{O}$, bonded $)$. \\
\hline $8 \mathbf{8}$ & $\begin{array}{l}392(19) \\
{\left[\mathrm{M}^{+}\right]}\end{array}$ & $3445(\mathrm{OH}), 1615(\mathrm{C}=\mathrm{C}), 1258(\mathrm{P}=\mathrm{O}), 1139,1080(\mathrm{P}-\mathrm{O}-\mathrm{C})$ \\
\hline $8 \mathbf{b}$ & $\begin{array}{l}392(30) \\
{\left[\mathrm{M}^{+}\right]}\end{array}$ & $3439(\mathrm{OH}), 1615(\mathrm{C}=\mathrm{C}), 1253(\mathrm{P}=\mathrm{O}), 1135,1079(\mathrm{P}-\mathrm{O}-\mathrm{C})$ \\
\hline $8 c$ & $\begin{array}{l}394(11) \\
{\left[\mathrm{M}^{+}\right]}\end{array}$ & $1620(\mathrm{C}=\mathrm{C}), 1249(\mathrm{P}=\mathrm{O}), 1138,1080(\mathrm{P}-\mathrm{O}-\mathrm{C})$ \\
\hline 8d & $\begin{array}{l}419(46) \\
{\left[\mathrm{M}^{+}\right]}\end{array}$ & $1623(\mathrm{C}=\mathrm{C}), 1237(\mathrm{P}=\mathrm{O}), 1140,1083(\mathrm{P}-\mathrm{O}-\mathrm{C})$ \\
\hline $8 \mathbf{e}$ & $\begin{array}{l}410(13) \\
{\left[\mathrm{M}^{+}\right]}\end{array}$ & $1628(\mathrm{C}=\mathrm{C}), 1240(\mathrm{P}=\mathrm{O}), 1138,1082(\mathrm{P}-\mathrm{O}-\mathrm{C})$ \\
\hline $8 f$ & $\begin{array}{l}410(17) \\
{\left[\mathrm{M}^{+}\right]}\end{array}$ & $1627(\mathrm{C}=\mathrm{C}), 1242(\mathrm{P}=\mathrm{O}), 1135,1083(\mathrm{P}-\mathrm{O}-\mathrm{C})$ \\
\hline 10 & $\begin{array}{l}516(13) \\
{\left[\mathrm{M}^{+}-1\right]}\end{array}$ & $\begin{array}{l}3450-3352(\mathrm{OH} \text { and } \mathrm{NH}), 1700,1685,1639(3 \times \mathrm{C}=\mathrm{O}), 1258(\mathrm{P}=\mathrm{O}), 1110 \\
(\mathrm{P}-\mathrm{O}-\mathrm{C})\end{array}$ \\
\hline
\end{tabular}


Table 3. ${ }^{1} \mathrm{H}-{ }^{31} \mathrm{P}$ - and ${ }^{13} \mathrm{C}$ - NMR data for compounds $6 \mathbf{6 a - j}, \mathbf{7}, \mathbf{8 a - f}$, and 10

\begin{tabular}{|c|c|c|}
\hline Comp & ${ }^{1} \mathrm{H}$ and ${ }^{31} \mathrm{P}$ NMR $\delta(\mathrm{ppm})$ & ${ }^{13} \mathrm{C}$ NMR $\delta(\mathrm{ppm})$ \\
\hline $6 \mathrm{a}^{\mathrm{a}}$ & $\begin{array}{l}1.58\left(\mathrm{dt}, J_{H H}=6.8,{ }^{4} J_{P H}=3.9 \mathrm{~Hz}, 12 \mathrm{H}, 4\right. \\
\left.\mathrm{CH}_{3}\right), 2.99\left(\mathrm{dd}, J_{H H}=9,{ }^{2} J_{P H}=13.7 \mathrm{~Hz}\right. \\
\left.1 \mathrm{H}, H^{a} \mathrm{C}\right), 3.87\left(\mathrm{ddd}, J_{H H}=9,11,{ }^{3} J_{P H}=\right. \\
\left.4.98 \mathrm{~Hz}, 1 \mathrm{H}, H^{b} \mathrm{C}\right), 4.25\left(\mathrm{qt}, J_{P H}=11.6\right. \\
\left.\mathrm{Hz}, 8 \mathrm{H}, 4 H_{2} \mathrm{CO}\right), 4.89\left(\mathrm{dd}, J_{H H}=11,{ }^{4} J_{P H}\right. \\
\left.=2.86 \mathrm{~Hz}, 1 \mathrm{H}, \mathrm{C} H^{\mathrm{c}}\right), 7.2-7.6(\mathrm{~m}, 4 \mathrm{H}, H- \\
\mathrm{Ar}), 8.47(\mathrm{~s}, 1 \mathrm{H}, p-\mathrm{O} H-\mathrm{Ar}), 10.1(2 \mathrm{~s}, 2 \mathrm{H}, \\
\left.2 H \mathrm{~N}^{\mathrm{c})}\right) ; \delta p:(2 \mathrm{~s}) 19.43,21.82 .\end{array}$ & $\begin{array}{l}16.1\left(\mathrm{t},{ }^{3} J_{P C}=3 \mathrm{~Hz}, 4 C \mathrm{H}_{3}\right), 35.8\left(\mathrm{t},{ }^{1} J_{P C}=\right. \\
\left.133 \mathrm{~Hz}, C-\mathrm{P}_{2}\right), 45.02\left(\mathrm{~d},{ }^{2} J_{P C}=7.2 \mathrm{~Hz},\right. \\
\left.C \mathrm{H}^{b}\right), 49.8\left(\mathrm{~d},{ }^{3} J_{P C}=4.5 \mathrm{~Hz}, C \mathrm{H}^{\mathrm{c}}\right), 62.2 \\
\left(\mathrm{t},{ }^{2} J_{C P}=6.7 \mathrm{~Hz}, 4 C \mathrm{CH}_{2} \mathrm{O}\right), 120.2,131.8, \\
132.8(C=C), 151.6(2-C=\mathrm{O}, \text { pyrimidine }) \\
154.5(\text { Ar- } C-\mathrm{OH}-p), 170.95,172.95(2 \mathrm{~d}, \\
4,6-C=\mathrm{O}, \text { pyrimidine }) .\end{array}$ \\
\hline $\mathbf{6 b}^{\mathrm{a}}$ & $\begin{array}{l}1.59\left(\mathrm{dt}, J_{H H}=7,{ }^{4} J_{P H}=4 \mathrm{~Hz}, 12 \mathrm{H}, 4\right. \\
\left.\mathrm{C}_{3}\right), 2.95\left(\mathrm{dd}, J_{H H}=8.7,{ }^{2} J_{P H}=13 \mathrm{~Hz},\right. \\
\left.1 \mathrm{H}, H^{a} \mathrm{C}\right), 3.92\left(\mathrm{ddd}, J_{H H}=9,11,{ }^{3} J_{P H}=\right. \\
\left.5.2 \mathrm{~Hz}, 1 \mathrm{H}, H^{b} \mathrm{C}\right), 4.126\left(\mathrm{qt}, J_{P H}=12 \mathrm{~Hz}\right. \\
\left.8 \mathrm{H}, 4 H_{2} \mathrm{CO}\right), 4.78(\mathrm{~s}, 1 \mathrm{H}, o-\mathrm{OH}-\mathrm{Ar}), 4.95 \\
\left(\mathrm{dd}, J_{H H}=11.2,{ }^{4} J_{P H}=2.7,1 \mathrm{H}, \mathrm{CH}{ }^{\mathrm{c}}\right), 7.2- \\
7.8(\mathrm{~m}, 4 \mathrm{H}, H-\mathrm{Ar}) ; \delta_{P}:(2 \mathrm{~s}) 20.1,22.6 .\end{array}$ & 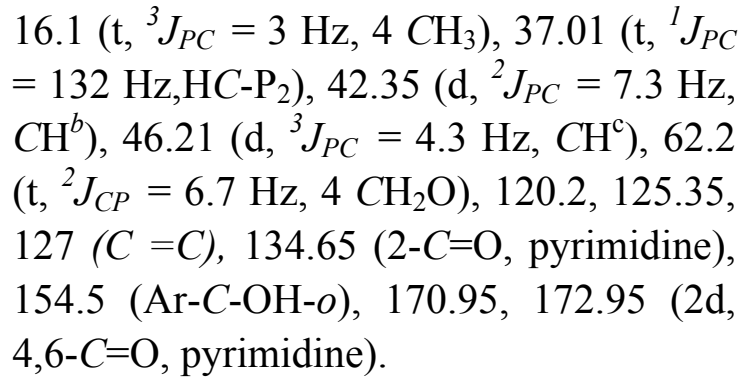 \\
\hline $6 c^{a}$ & $\begin{array}{l}1.56\left(\mathrm{dt}, J_{H H}=6.9,{ }^{4} J_{P H}=3.9 \mathrm{~Hz}, 12 \mathrm{H}, 4\right. \\
\left.\mathrm{C}_{3}\right), 2.89\left(\mathrm{dd}, J_{H H}=8.6,{ }^{2} J_{P H}=13.5 \mathrm{~Hz},\right. \\
\left.1 \mathrm{H}, H^{a} \mathrm{C}\right), 3.95\left(\mathrm{ddd}, J_{H H}=9,11,{ }^{3} J_{P H}=6\right. \\
\left.\mathrm{Hz}, 1 \mathrm{H}, H^{b} \mathrm{C}\right), 4.24\left(\mathrm{qt}, J_{P H}=11.9 \mathrm{~Hz}, 8 \mathrm{H},\right. \\
\left.4 H_{2} \mathrm{CO}\right), 4.88\left(\mathrm{dd}, J_{H H}=11 \mathrm{~Hz},{ }^{4} J_{P H}=2.5\right. \\
\left.\mathrm{Hz}, 1 \mathrm{H}, H^{c} \mathrm{C}\right), 7.3-7.9(\mathrm{~m}, 4 \mathrm{H}, H-\mathrm{Ar}) ; \delta_{P}: \\
(2 \mathrm{~s}) 21.68,23.01\end{array}$ & $\begin{array}{l}16.1\left(\mathrm{t},{ }^{3} J_{P C}=3 \mathrm{~Hz}, 4 C \mathrm{H}_{3}\right), 35.8\left(\mathrm{t},{ }^{1} J_{P C}=\right. \\
\left.132.7 \mathrm{~Hz}, C-\mathrm{P}_{2}\right), 45.02\left(\mathrm{~d},{ }^{2} J_{P C}=7.2 \mathrm{~Hz},\right. \\
\left.C \mathrm{H}^{b}\right), 49.9\left(\mathrm{~d},{ }^{3} J_{P C}=4.6 \mathrm{~Hz}, C \mathrm{H}^{\mathrm{c}}\right), 62.2 \\
\left(\mathrm{t},{ }^{2} J_{C P}=6.7 \mathrm{~Hz}, 4 C \mathrm{H}_{2} \mathrm{O}\right), 118.5,119.2, \\
133,141.3 \quad(C=C), 150.1 \quad((2-C=\mathrm{O}, \\
\text { pyrimidine }), 163.63 \text { (Ar- } C-\mathrm{F}-p), 170.92, \\
172.95(2 \mathrm{~d}, 4,6-C=\mathrm{O}, \text { pyrimidine). }\end{array}$ \\
\hline $6 d^{\mathrm{a}}$ & $\begin{array}{l}1.58\left(\mathrm{dt}, J_{H H}=6.7,{ }^{4} J_{P H}=3.8 \mathrm{~Hz}, 12 \mathrm{H}, 4\right. \\
\left.\mathrm{C}_{3}\right), 2.5\left(2 \mathrm{~s}, 6 \mathrm{H}, \mathrm{N}\left(\mathrm{CH}_{3}\right)_{2}\right), 2.97\left(\mathrm{dd}, J_{H H}\right. \\
\left.=8.8,{ }^{2} J_{P H}=13.8 \mathrm{~Hz}, 1 \mathrm{H}, H^{a} \mathrm{C}\right), 3.57(\mathrm{ddd}, \\
\left.J_{H H}=9,11,{ }^{3} J_{P H}=4.99 \mathrm{~Hz}, 1 \mathrm{H}, H^{b} \mathrm{C}\right), \\
4.23\left(\mathrm{qt}, J_{P H}=12 \mathrm{~Hz}, 8 \mathrm{H}, 4 H_{2} \mathrm{CO}\right), 4.95 \\
\left(\mathrm{dd}, J_{H H}=10.8,{ }^{4} J_{P H}=2.9 \mathrm{~Hz}, 1 \mathrm{H}, H^{c} \mathrm{C}\right), \\
7.42-8.01(\mathrm{~m}, 4 \mathrm{H}, H-\mathrm{Ar}) ; \delta_{P}:(2 \mathrm{~s}) 19.5, \\
21.82 .\end{array}$ & $\begin{array}{l}16.1\left(\mathrm{t},{ }^{3} J_{P C}=2.8 \mathrm{~Hz}, 4 \mathrm{CH}_{3}\right), 35.8\left(\mathrm{t},{ }^{l} J_{P C}\right. \\
\left.=132.7 \mathrm{~Hz}, C-\mathrm{P}_{2}\right), 39.05\left(\mathrm{~d},{ }^{2} J_{P C}=7.2 \mathrm{~Hz},\right. \\
\left.C \mathrm{H}^{b}\right), 40.5\left(\mathrm{~N}\left(C \mathrm{H}_{3}\right)_{2-} \mathrm{Ar}\right) 49.9\left(\mathrm{~d},{ }^{3} J_{P C}=\right. \\
\left.4.6 \mathrm{~Hz}, C \mathrm{H}^{\mathrm{c}}\right), 62.1\left(\mathrm{t},{ }^{2} J_{C P}=6.7 \mathrm{~Hz}, 4\right. \\
\left.C \mathrm{H}_{2} \mathrm{O}\right), 119,120.2,132.3,148.4(C=C), \\
150.7((2-C=\mathrm{O}, \text { pyrimidine }), 171,172.95 \\
(2 \mathrm{~d}, 4,6-C=\mathrm{O}, \text { pyrimidine }) .\end{array}$ \\
\hline $6 \mathrm{e}^{\mathrm{a}}$ & $\begin{array}{l}1.58\left(\mathrm{dt}, J_{H H}=6.5,{ }^{4} J_{P H}=3.8 \mathrm{~Hz}, 12 \mathrm{H}, 4\right. \\
\left.\mathrm{C} H_{3}\right), 2.93\left(\mathrm{~d} \text { of d, } J_{H H}=8.6,{ }^{2} J_{P H}=13.2\right. \\
\left.\mathrm{Hz}, 1 \mathrm{H}, H^{a} \mathrm{C}\right), 3.98\left(\mathrm{ddd}, J_{H H}=9,11,{ }^{3} J_{P H}\right. \\
\left.=4.7 \mathrm{~Hz}, 1 \mathrm{H}, H^{b} \mathrm{C}\right), 4.25\left(\mathrm{qt}, J_{P H}=12 \mathrm{~Hz}\right. \\
\left.8 \mathrm{H}, 4 H_{2} \mathrm{CO}\right), 4.89\left(\mathrm{dd},{ }^{4} J_{P H}=2.8 \mathrm{~Hz}, 1 \mathrm{H},\right. \\
\left.\mathrm{C} H^{c}\right), 7.2-7.7(\mathrm{~m}, 4 \mathrm{H}, H-\mathrm{Ar}) ; \delta_{P}: 20.1, \\
23.02 .\end{array}$ & $\begin{array}{l}16.1\left(\mathrm{t},{ }^{3} J_{P C}=3.1 \mathrm{~Hz}, 4 \mathrm{CH}_{3}\right), 36.8\left(\mathrm{t},{ }^{1} J_{P C}\right. \\
\left.=132.4 \mathrm{~Hz}, C-\mathrm{P}_{2}\right), 46.05\left(\mathrm{~d},{ }^{2} J_{P C}=6.8 \mathrm{~Hz},\right. \\
\left.C^{b} \mathrm{H}^{b}\right), 47.5\left(\mathrm{~d},{ }^{3} J_{P C}=4.2 \mathrm{~Hz}, C \mathrm{H}^{\mathrm{c}}\right), 62.1(\mathrm{t}, \\
\left.{ }^{2} J_{C P}=6.7 \mathrm{~Hz}, 4 C \mathrm{H}_{2} \mathrm{O}\right), 127.1,130.8,134, \\
136,137.7 \quad(C=C), 152.7 \quad((2-C=\mathrm{O}, \\
\text { pyrimidine }), 169.1,171 \quad(2 \mathrm{~d}, 4,6-C=\mathrm{O}, \\
\text { pyrimidine). }\end{array}$ \\
\hline $6 f^{a}$ & $\begin{array}{l}1.58\left(\mathrm{dt}, J_{H H}=6.4, J_{P H}=3\right. \\
\left.\mathrm{CH}_{3}\right), 2.91\left(\mathrm{dd}, J_{H H}=8.6,\right.\end{array}$ & $\begin{array}{l}16.3\left(\mathrm{t},{ }^{3} J_{P C}=3.2 \mathrm{~Hz}, 4 C_{3}\right), 35.6\left(\mathrm{t},{ }^{1} J_{P C}\right. \\
\left.=132.1 \mathrm{~Hz}, C-\mathrm{P}_{2}\right), 41.05\left(\mathrm{~d},{ }^{2} J_{P C}=7.2 \mathrm{~Hz},\right.\end{array}$ \\
\hline
\end{tabular}


$\left.1 \mathrm{H}, H^{a} \mathrm{C}\right), 3.98\left(\mathrm{ddd}, J_{H H}=9,11,{ }^{3} J_{P H}=\right.$ $5.4 \mathrm{~Hz}, 1 \mathrm{H}, H^{b} \mathrm{C}$ ), 4.05 (qt, $J_{P H}=12.1 \mathrm{~Hz}$, $\left.8 \mathrm{H}, 4 \mathrm{H}_{2} \mathrm{CO}\right), 4.81\left(\mathrm{dd}, J_{H H}=11,{ }^{4} J_{P H}=\right.$ $\left.2.6 \mathrm{~Hz}, 1 \mathrm{H}, H^{c} \mathrm{C}\right), 7.58-7.68(\mathrm{~m}, 4 \mathrm{H}, H-$ $\mathrm{Ar}) ; \delta_{P}:(2 \mathrm{~s}) 21.7,23.2$.

$\mathbf{6 g}^{\mathrm{a}} \quad 1.61\left(\mathrm{dt}, J_{H H}=6.7,{ }^{4} J_{P H}=3.8 \mathrm{~Hz}, 12 \mathrm{H}, 4\right.$ $\left.\mathrm{CH}_{3}\right), 2.89\left(\mathrm{dd}, J_{H H}=8.8,{ }^{2} J_{P H}=13.8 \mathrm{~Hz}\right.$, $\left.1 \mathrm{H}, H^{a} \mathrm{C}\right), 3.15\left(2 \mathrm{~s}, 6 \mathrm{H}, 2 \mathrm{NCH}_{3}\right), 3.98$ $\left(\mathrm{ddd}, J_{H H}=9,11,{ }^{3} J_{P H}=4.99 \mathrm{~Hz}, 1 \mathrm{H}\right.$, $H^{b} \mathrm{C}$ ), 4.27 (qt, $J_{P H}=12 \mathrm{~Hz}, 8 \mathrm{H}, 4 \mathrm{H}_{2} \mathrm{CO}$ ), $4.95\left(\mathrm{dd}, J_{H H}=10.8,{ }^{4} J_{P H}=2.8 \mathrm{~Hz}, 1 \mathrm{H}\right.$, $\left.H^{c} \mathrm{C}\right), 7.32-7.71(\mathrm{~m}, 4 \mathrm{H}, H-\mathrm{Ar}), 8.47(\mathrm{~s}, 1 \mathrm{H}$, p-OH-Ar); $\delta_{P}:(2 \mathrm{~s}) 19.5,21.82$.

$\mathbf{6 h}^{\mathrm{a}} \quad 1.61\left(\mathrm{dt}, J_{H H}=6.4,{ }^{4} J_{P H}=3.8 \mathrm{~Hz}, 12 \mathrm{H}, 4\right.$ $\left.\mathrm{CH}_{3}\right), 2.89\left(\mathrm{dd}, J_{H H}=8.4,{ }^{2} J_{P H}=13.8 \mathrm{~Hz}\right.$, $\left.1 \mathrm{H}, H^{a} \mathrm{C}\right), 3.15\left(2 \mathrm{~s}, 6 \mathrm{H}, 2 \mathrm{NCH}_{3}\right), 3.97$ $\left(\mathrm{ddd}, J_{H H}=9,11,{ }^{3} J_{P H}=4.3 \mathrm{~Hz}, 1 \mathrm{H}\right.$, $H^{b} \mathrm{C}$ ), 4.17 (qt, $J_{P H}=11.8 \mathrm{~Hz}, 8 \mathrm{H}, 4$ $\mathrm{H}_{2} \mathrm{CO}$ ), 4.77 (s, 1H, o-OH-Ar), 4.95 (dd, $\left.J_{H H}=11.1,{ }^{4} J_{P H}=2.5 \mathrm{~Hz}, 1 \mathrm{H}, H^{c} \mathrm{C}\right), 7.32-$ 7.81(m, 4H, $H$-Ar); $\delta_{P}$ : (2s) 20.5, 22.82 $\mathbf{6 i}^{\mathrm{b}} \quad 1.56\left(\mathrm{dt}, J_{H H}=6.8,{ }^{4} J_{P H}=3.4 \mathrm{~Hz}, 12 \mathrm{H}, 4\right.$ $\left.\mathrm{CH}_{3}\right), 2.97\left(\mathrm{dd}, J_{H H}=8.4,{ }^{2} J_{P H}=13.8 \mathrm{~Hz}\right.$, $\left.1 \mathrm{H}, H^{a} \mathrm{C}\right), 3.15\left(2 \mathrm{~s}, 6 \mathrm{H}, 2 \mathrm{NCH}_{3}\right), 3.68$ $\left(\mathrm{ddd}, J_{H H}=9,11,{ }^{3} J_{P H}=4.8 \mathrm{~Hz}, 1 \mathrm{H}\right.$, $H^{b} \mathrm{C}$ ), 4.21 (qt, $J_{P H}=11.9 \mathrm{~Hz}, 8 \mathrm{H}, 4$ $\mathrm{H}_{2} \mathrm{CO}$ ), 4.92 (s, 1H, o-OH-Ar), 4.95 (dd, $\left.J_{H H}=11.1,{ }^{4} J_{P H}=2.8 \mathrm{~Hz}, 1 \mathrm{H}, H^{c} \mathrm{C}\right), 7.5-$ 8.1(m, 4H, H-Ar); $\delta_{P}:(2 \mathrm{~s}) 20.19,21.9$. $\mathbf{6 j}^{\mathrm{b}} \quad 1.6\left(\mathrm{dt}, J_{H H}=6.7,{ }^{4} J_{P H}=3.7 \mathrm{~Hz}, 12 \mathrm{H}, 4\right.$ $\left.\mathrm{CH}_{3}\right), 2.45\left(2 \mathrm{~s}, 6 \mathrm{H}, \mathrm{N}\left(\mathrm{CH}_{3}\right)_{2}\right), 2.92(\mathrm{dd}$, $\left.J_{H H}=8.5,{ }^{2} J_{P H}=13.9 \mathrm{~Hz}, 1 \mathrm{H}, H^{a} \mathrm{C}\right), 3.15$ $\left(2 \mathrm{~s}, 6 \mathrm{H}, 2 \mathrm{NCH}_{3}\right), 3.81$ (ddd, $J_{H H}=9,11$, $\left.{ }^{3} J_{P H}=5.2 \mathrm{~Hz}, 1 \mathrm{H}, H^{b} \mathrm{C}\right), 4.27\left(\mathrm{qt}, J_{P H}=\right.$ $\left.12.2 \mathrm{~Hz}, 8 \mathrm{H}, 4 \mathrm{H}_{2} \mathrm{CO}\right), 4.96\left(\mathrm{dd}, J_{H H}=\right.$ $\left.10.81,{ }^{4} J_{P H}=2.9 \mathrm{~Hz}, 1 \mathrm{H}, H^{c} \mathrm{C}\right), 7.42-$ 8.1(m, 4H, H-Ar); $\delta_{P}$ : (2s) 20.59, 22.13. $2.95\left(\mathrm{dd}, J_{H H}=10.5,{ }^{2} J_{P H}=12.6 \mathrm{~Hz}, 1 \mathrm{H}\right.$, $\left.H C-\mathrm{P}_{2}\right), 3.17$ (2s, $\left.6 \mathrm{H}, \mathrm{N}\left(\mathrm{CH}_{3}\right)_{2}\right), 3.91$ (ddd, $\left.J_{H H}=9,11,{ }^{3} J_{P H}=4 \mathrm{~Hz}, 1 \mathrm{H}, H^{b} \mathrm{C}\right), 4.12$ $\left(\mathrm{dd}, J_{H H}=11 \mathrm{~Hz},{ }^{3} J_{P H}=3.8 \mathrm{~Hz}, 1 \mathrm{H}, H^{c} \mathrm{C}\right)$,
$\left.C \mathrm{H}^{b}\right), 49.3\left(\mathrm{~d},{ }^{3} J_{P C}=4.5 \mathrm{~Hz}, C \mathrm{H}^{\mathrm{c}}\right), 62.1(\mathrm{t}$, $\left.{ }^{2} J_{C P}=6.8 \mathrm{~Hz}, 4 \mathrm{CH}_{2} \mathrm{O}\right), 126.1,130.1$, $1342.9,136.1,139.7 \quad(C=C), 152.7$ ((2$C=\mathrm{O}$, pyrimidine), 170.3, 173.7 (2d, 4,6$C=\mathrm{O}$, pyrimidine).

$16.1\left(\mathrm{t},{ }^{3} \mathrm{~J}_{P C}=3 \mathrm{~Hz}, 4 \mathrm{CH}_{3}\right), 28.3\left(2 \mathrm{NCH}_{3}\right.$, pyrimidine), $35.5\left(\mathrm{t},{ }^{1} J_{P C}=132.1 \mathrm{~Hz}, C\right.$ $\left.\mathrm{P}_{2}\right), 39.5\left(\mathrm{~d},{ }^{2} J_{P C}=7.2 \mathrm{~Hz}, C \mathrm{H}^{b}\right), 52.2(\mathrm{~d}$, $\left.{ }^{3} J_{P C}=4.2 \mathrm{~Hz}, C \mathrm{H}^{\mathrm{c}}\right), 62.3\left(\mathrm{t},{ }^{2} J_{C P}=6.9 \mathrm{~Hz}\right.$, $\left.4 \mathrm{CH}_{2} \mathrm{O}\right), 119.5,132.3,143.6(\mathrm{C}=C)$, $152.7((2-C=\mathrm{O}$, pyrimidine), 154.3 (Ar- $C$ $\mathrm{OH}-p), \quad 165.9, \quad 166.9 \quad(2 \mathrm{~d}, \quad 4,6-C=\mathrm{O}$, pyrimidine).

$16.1\left(\mathrm{t},{ }^{3} J_{P C}=3.2 \mathrm{~Hz}, 4 \mathrm{CH}_{3}\right), 28.5$ $\left(2 \mathrm{NCH}_{3}\right.$, pyrimidine), $39.5\left(\mathrm{t},{ }^{1} J_{P C}=134.1\right.$ $\left.\mathrm{Hz}, C-\mathrm{P}_{2}\right), 42.5\left(\mathrm{~d},{ }^{2} J_{P C}=7.2 \mathrm{~Hz}, C \mathrm{H}^{b}\right)$, $45.2\left(\mathrm{~d},{ }^{3} J_{P C}=4.1 \mathrm{~Hz}, C \mathrm{H}^{\mathrm{c}}\right), 62.1\left(\mathrm{t},{ }^{2} J_{C P}=\right.$ $\left.6.6 \mathrm{~Hz}, 4 \mathrm{CH}_{2} \mathrm{O}\right), 118.5,125.3,126.1$, 129.5, $133.3 \quad(C=C), 151.1 \quad(2-C=\mathrm{O}$, pyrimidine), 157.3 (Ar- $C-\mathrm{OH}-o$ ), 166.9, 168.7 (2d, 4,6- $C=\mathrm{O}$, pyrimidine).

$16.1\left(\mathrm{t},{ }^{3} J_{P C}=3 \mathrm{~Hz}, 4 \mathrm{CH}_{3}\right), 28.2\left(2 \mathrm{NCH}_{3}\right.$, pyrimidine), $35.5\left(\mathrm{t},{ }^{1} J_{P C}=132.1 \mathrm{~Hz}, C\right.$ $\left.\mathrm{P}_{2}\right), 39.5\left(\mathrm{~d},{ }^{2} J_{P C}=7.1 \mathrm{~Hz}, C \mathrm{H}^{b}\right), 40.5$ $\left(\mathrm{N}\left(\mathrm{CH}_{3}\right)_{2} \mathrm{Ar}\right), \quad 52.2\left(\mathrm{~d},{ }^{3} J_{P C}=4.6 \mathrm{~Hz}\right.$, $\left.\mathrm{CH}^{\mathrm{c}}\right), 62.1\left(\mathrm{t},{ }^{2} J_{C P}=6.7 \mathrm{~Hz}, 4 \mathrm{CH}_{2} \mathrm{O}\right)$, 116.5, 133.3, $140.6(C=C), 152.1((2-C=\mathrm{O}$, pyrimidine), 166.9, $168.9(2 \mathrm{~d}, 4,6-C=\mathrm{O}$, pyrimidine).

$16.2\left(\mathrm{t},{ }^{3} J_{P C}=2.9 \mathrm{~Hz}, 4 \mathrm{CH}_{3}\right), 28.2$ $\left(2 \mathrm{NCH}_{3}\right.$, pyrimidine), $36.2\left(\mathrm{t},{ }^{1} J_{P C}=132.7\right.$ $\left.\mathrm{Hz}, C-\mathrm{P}_{2}\right), 39.05\left(\mathrm{~d},{ }^{2} J_{P C}=7.3 \mathrm{~Hz}, C \mathrm{H}^{b}\right)$, $41.3\left(\mathrm{~N}\left(\mathrm{CH}_{3}\right)_{2}-\mathrm{Ar}\right), 52.2\left(\mathrm{~d},{ }^{3} J_{P C}=4.6 \mathrm{~Hz}\right.$, $\left.\mathrm{CH}^{\mathrm{c}}\right), 62.1\left(\mathrm{t},{ }^{2} J_{C P}=6.7 \mathrm{~Hz}, 4 \mathrm{CH}_{2} \mathrm{O}\right)$, 116.5, 133.3, $148.6(C=C), 152.1((2-C=\mathrm{O}$, pyrimidine), $166.9,168.95(2 \mathrm{~d}, 4,6-C=\mathrm{O}$, pyrimidine).

$227.8\left(2 \mathrm{NCH}_{3}\right.$, pyrimidine), $39.5\left(\mathrm{t},{ }^{1} J_{P C}=\right.$ $\left.134.1 \mathrm{~Hz}, C-\mathrm{P}_{2}\right), 41.5\left(\mathrm{~d},{ }^{2} J_{P C}=7.2 \mathrm{~Hz}\right.$, $\left.C \mathrm{H}^{b}\right), 44.6\left(\mathrm{~d},{ }^{3} J_{P C}=4.1 \mathrm{~Hz}, C \mathrm{H}^{\mathrm{c}}\right), 119.5$, $125.3,126.1,129.5,136.3(C=C), 151.1$ 
7.44-8.1 (m, 4 H, H-Ar). $\delta_{P}$ : (2s) 20.3, 22.9

$8 a^{a}$

$1.23,1.36\left(2 \mathrm{dt}, J_{H H}=7 \mathrm{~Hz},{ }^{4} J_{P H}=4.5 \mathrm{~Hz}\right.$, $\left.\mathrm{Hz}, 12 \mathrm{H}, 4 \mathrm{H}_{3} \mathrm{C}\right), 3.96-4.17$ (m, $8 \mathrm{H}, 4$ $\mathrm{H}_{2} \mathrm{CO}$ ), 7.23-7.58 (m, 4H, H-Ar), 8.1 (dd, $\left.{ }^{3} J_{P H}=6.2 \mathrm{~Hz}, 1 \mathrm{H}, H \mathrm{C}=\mathrm{C}-\mathrm{P}_{2}\right), 8.47(\mathrm{~s}, 1 \mathrm{H}$, $p$-OH-Ar); $\delta_{P}$ : (2s) 21.31, 23.92.

$\mathbf{8 b}^{\mathrm{a}} \quad 1.15,1.41\left(2 \mathrm{dt}, J_{H H}=6.8,{ }^{4} J_{P H}=4.6 \mathrm{~Hz}, 12\right.$ $\left.\mathrm{H}, 4 \mathrm{H}_{3} \mathrm{C}\right), 3.98-4.16\left(\mathrm{~m}, 8 \mathrm{H}, 4 \mathrm{H}_{2} \mathrm{CO}\right)$, $4.78(\mathrm{~s}, 1 \mathrm{H}, o-\mathrm{OH}-\mathrm{Ar}), 7.21-7.78(\mathrm{~m}, 4 \mathrm{H}$, $H$-Ar), $8.36\left(\mathrm{dd},{ }^{3} J_{P H}=6.2 \mathrm{~Hz}, 1 \mathrm{H}\right.$, $\left.H \mathrm{C}=\mathrm{C}-\mathrm{P}_{2}\right) ; \delta_{P}$ : (2s) 21.63, 23.2.

$\mathbf{8 c}^{\mathrm{b}} \quad 1.16,1.25\left(2 \mathrm{dt}, J_{H H}=6.7,{ }^{4} J_{P H}=4.5 \mathrm{~Hz}, 12\right.$ $\left.\mathrm{H}, 4 \mathrm{H}_{3} \mathrm{C}\right), 3.96-4.17\left(\mathrm{~m}, 8 \mathrm{H}, 4 \mathrm{H}_{2} \mathrm{CO}\right)$, 7.31-7.78 (m, 4H, H-Ar), 8.31 (dd, ${ }^{3} J_{P H}=$ $\left.6.2 \mathrm{~Hz}, 1 \mathrm{H}, H \mathrm{C}=\mathrm{C}-\mathrm{P}_{2}\right) ; \delta_{P}:(2 \mathrm{~s}) 22.3,23.2$.

$\mathbf{8 d}^{\mathrm{b}} \quad 1.15,1.28\left(2 \mathrm{dt}, J_{H H}=6.5,{ }^{4} J_{P H}=4.6 \mathrm{~Hz}, 12\right.$ $\left.\mathrm{H}, 4 \mathrm{H}_{3} \mathrm{C}\right), 2.57\left(2 \mathrm{~s}, 6 \mathrm{H}, \mathrm{N}\left(\mathrm{CH}_{3}\right)_{2}\right), 3.95-$ $4.13\left(\mathrm{~m}, 8 \mathrm{H}, 4 \mathrm{H}_{2} \mathrm{CO}\right), 6.75-7.78(\mathrm{~m}, 4 \mathrm{H}$, $H$-Ar), $8.18\left(\mathrm{dd},{ }^{3} J_{P H}=6.8 \mathrm{~Hz}, 1 \mathrm{H}\right.$, $\left.H \mathrm{C}=\mathrm{C}-\mathrm{P}_{2}\right) ; \delta_{P}:(2 \mathrm{~s}) 22.63,23.1$.

$\mathbf{8 e}^{\mathrm{b}} \quad 1.13,1.25\left(2 \mathrm{dt}, J_{H H}=6.3,{ }^{4} J_{P H}=4.5 \mathrm{~Hz}, 12\right.$ $\left.\mathrm{H}, 4 \mathrm{H}_{3} \mathrm{C}\right), 3.98-4.14\left(\mathrm{~m}, 8 \mathrm{H}, 4 \mathrm{H}_{2} \mathrm{CO}\right)$, 7.29-7.46 (m, 4H, H-Ar), $8.35\left(\mathrm{dd},{ }^{3} J_{P H}=\right.$ $\left.6.8 \mathrm{~Hz}, 1 \mathrm{H}, H \mathrm{C}=\mathrm{C}-\mathrm{P}_{2}\right) ; \delta_{P}:(2 \mathrm{~s}) 21.8,22.9$. $\mathbf{8 f}^{\mathrm{b}} \quad 1.16,1.27\left(2 \mathrm{dt}, J_{H H}=6.7,{ }^{4} J_{P H}=4.2 \mathrm{~Hz}, 12\right.$ $\left.\mathrm{H}, 4 \mathrm{H}_{3} \mathrm{C}\right), 3.96-4.17\left(\mathrm{~m}, 8 \mathrm{H}, 4 \mathrm{H}_{2} \mathrm{CO}\right)$, 7.35-7.75 (m, 4H, H-Ar), 8.25 (dd, ${ }^{3} J_{P H}=$ $\left.6.6 \mathrm{~Hz}, 1 \mathrm{H}, H \mathrm{C}=\mathrm{C}-\mathrm{P}_{2}\right)$; $\delta_{P}$ : (2s) 22.1 , 23.02 .

$10^{\mathrm{a}}$ $1.35\left(\mathrm{dt}, J_{H H}=6.8,{ }^{4} J_{P H}=2.8 \mathrm{~Hz}, 6 \mathrm{H}, 2\right.$ $\left.\mathrm{CH}_{3}\right), 3.87\left(\mathrm{dq}, J_{H H}=6.5,{ }^{3} J_{P H}=4.7 \mathrm{~Hz}\right.$, $\left.4 \mathrm{H}, 2 \mathrm{H}_{2} \mathrm{CO}\right), 4.41$ (d, ${ }^{3} J_{P H}=4.95 \mathrm{~Hz}, 1 \mathrm{H}$, $\left.H^{\mathrm{b}} \mathrm{C}-\mathrm{C}-\mathrm{P}\right), 5.45\left(\mathrm{~d},{ }^{4} J_{P H}=2.3 \mathrm{~Hz}, 1 \mathrm{H}, H^{c} \mathrm{C}\right.$, pyrimidine) $6.89\left(\mathrm{~d},{ }^{2} J_{P H}=15.4 \mathrm{~Hz}, 1 \mathrm{H}\right.$, $=H \mathrm{C}), 7.26-8.3(\mathrm{~m}, 8 \mathrm{H}, H$-Ar $), 8.59(\mathrm{~s}, 1 \mathrm{H}$, $p$-OH-Ar), $10.1\left(2 \mathrm{~s}, 2 \mathrm{H}, 2 H \mathrm{~N}^{\mathrm{c})}\right) ; \delta p:(2 \mathrm{~s})$ 20.8 .
(2- $C=\mathrm{O}$, pyrimidine), 157.8 (Ar- $C-\mathrm{OH}-o$ ), $166.5,168.8(2 \mathrm{~d}, 4,6-C=\mathrm{O}$, pyrimidine).

$16.3\left(\mathrm{t},{ }^{3} J_{P C}=3.2 \mathrm{~Hz}, 4 \mathrm{CH}_{3}\right), 62.2\left(\mathrm{t},{ }^{2} J_{C P}\right.$ $\left.=6.7 \mathrm{~Hz}, 4 \mathrm{CH}_{2} \mathrm{O}\right), 120.2,129.8,134.8(\mathrm{C}$ =C), $127.1\left(\mathrm{t},{ }^{1} J_{P C}=133.5 \mathrm{~Hz}, C-\mathrm{P}_{2}\right)$, 158.6 (Ar- $C$-OH- $p$ ), 168.05 (t, ${ }^{2} J_{C P}=7.3$ $\left.\mathrm{Hz}, \mathrm{HC}=\mathrm{C}-\mathrm{P}_{2}\right)$.

$16.2\left(\mathrm{t},{ }^{3} J_{P C}=3.4 \mathrm{~Hz}, 4 \mathrm{CH}_{3}\right), 62.7\left(\mathrm{t},{ }^{2} J_{C P}\right.$ $\left.=7 \mathrm{~Hz}, 4 \mathrm{CH}_{2} \mathrm{O}\right), 126.2,128.8,133.8(\mathrm{C}$ $=C), 129.1\left(\mathrm{t},{ }^{1} J_{P C}=132.8 \mathrm{~Hz}, C-\mathrm{P}_{2}\right)$, 156.3 (Ar-C-OH-o), $166.6\left(\mathrm{t},{ }^{2} J_{C P}=7.1\right.$ $\left.\mathrm{Hz}, \mathrm{HC}=\mathrm{C}-\mathrm{P}_{2}\right)$.

$16.2\left(\mathrm{t},{ }^{3} J_{P C}=3.1 \mathrm{~Hz}, 4 \mathrm{CH}_{3}\right), 62.5\left(\mathrm{t},{ }^{2} J_{C P}\right.$ $\left.=7 \mathrm{~Hz}, 4 \mathrm{CH}_{2} \mathrm{O}\right), 119.2,126.8,134.8(\mathrm{C}$ $=C), 127.1\left(\mathrm{t},{ }^{1} J_{P C}=133.4 \mathrm{~Hz}, C-\mathrm{P}_{2}\right)$, $164.1\left(\right.$ Ar- $C$-F- $p$ ), $165.8\left(\mathrm{t},{ }^{2} J_{C P}=7.1 \mathrm{~Hz}\right.$, $\left.\mathrm{HC}=\mathrm{C}-\mathrm{P}_{2}\right)$.

$16.3\left(\mathrm{t},{ }^{3} J_{P C}=2.8 \mathrm{~Hz}, 4 \mathrm{CH}_{3}\right), 39.6$ $\left(\mathrm{N}\left(\mathrm{CH}_{3}\right)_{2} \mathrm{Ar}\right), 62.4\left(\mathrm{t},{ }^{2} J_{C P}=6.8 \mathrm{~Hz}, 4\right.$ $\left.\mathrm{CH}_{2} \mathrm{O}\right), 116.2,127.8,130.8(\mathrm{C}=\mathrm{C}), 126.8$ $\left(\mathrm{t},{ }^{l} J_{P C}=133.8 \mathrm{~Hz}, C-\mathrm{P}_{2}\right), 151.08(C-$ $\left.\mathrm{N}\left(\mathrm{CH}_{3}\right)_{2}-\mathrm{Ar}\right), 166.05\left(\mathrm{t},{ }^{2} J_{C P}=6.9 \mathrm{~Hz}\right.$, $\left.\mathrm{HC}=\mathrm{C}-\mathrm{P}_{2}\right)$.

$16.3\left(\mathrm{t},{ }^{3} J_{P C}=3.4 \mathrm{~Hz}, 4 \mathrm{CH}_{3}\right), 62.5\left(\mathrm{t},{ }^{2} J_{C P}\right.$ $\left.=7.2 \mathrm{~Hz}, 4 \mathrm{CH}_{2} \mathrm{O}\right), 126.7\left(\mathrm{t},{ }^{1} J_{P C}=132.8\right.$ $\left.\mathrm{Hz}, C-\mathrm{P}_{2}\right), 130.6,133.8,137.2(C=C)$, $161.4\left(\mathrm{t},{ }^{2} J_{C P}=7.1 \mathrm{~Hz}, \mathrm{HC}=\mathrm{C}-\mathrm{P}_{2}\right)$.

$16.3\left(\mathrm{t},{ }^{3} J_{P C}=3.1 \mathrm{~Hz}, 4 \mathrm{CH}_{3}\right), 62.1\left(\mathrm{t},{ }^{2} J_{C P}\right.$ $\left.=6.9 \mathrm{~Hz}, 4 \mathrm{CH}_{2} \mathrm{O}\right), 126.9\left(\mathrm{t},{ }^{1} J_{P C}=133.2\right.$ $\left.\mathrm{Hz}, C-\mathrm{P}_{2}\right), 129.9,132.8,141.2(C=C)$, $165.8\left(\mathrm{t},{ }^{2} J_{C P}=7.4 \mathrm{~Hz}, \mathrm{HC}=\mathrm{C}-\mathrm{P}_{2}\right)$.

$16.2\left(\mathrm{~d},{ }^{3} J_{P C}=3.7 \mathrm{~Hz}, 2 \mathrm{CH}_{3}\right), 45.8(\mathrm{~d}$, $\left.{ }^{2} J_{P C}=7.5 \mathrm{~Hz}, C \mathrm{H}^{\mathrm{b}}-\mathrm{C}-\mathrm{P}\right), 51.5 \quad\left(\mathrm{~d},{ }^{3} J_{P C}=\right.$ $\left.4.5 \mathrm{~Hz}, C^{\mathrm{c}}\right), 62.1\left(\mathrm{t},{ }^{2} J_{C P}=7.2 \mathrm{~Hz}, 2\right.$ $\left.\mathrm{CH}_{2} \mathrm{O}\right), 118.1,122.8,129.1,130.2,138$, $145.1(C=C), 152.6$ (2- $C=\mathrm{O}$, pyrimidine), 156.5 (Ar- $C-\mathrm{OH}-p), 170.6,172.4(2 \mathrm{~d}, 4,6-$ $\mathrm{C}=\mathrm{O}$, pyrimidine).

* Solvents for NMR: ${ }^{\mathrm{a}} \mathrm{DMSO}-\mathrm{d}_{6} ;{ }^{\mathrm{b}} \mathrm{CDCl}_{3} ;{ }^{\mathrm{c}}$ Proton exchangeable with $\mathrm{D}_{2} \mathrm{O}$. 


\section{Pharmacological evaluation}

The effect on bone resorption of these new compounds was investigated in terms of the structure activity relationships (SAR) of BPs. The screening relied on assessing the potency in an in vivo bone resorption model. Compounds $\mathbf{6 a - j}$ and 7 were tested in thyroparathyroidectomized (TPTX) rats with hypercalcemia induced by 1,25 -dihydroxy vitamin $\mathrm{D}_{3},{ }^{15}$ by analogy to the activity of risedronate as a reference. BP-risedronate ( $c f$. Fig 2) was selected for its optimal potency and safety in early screening assays. ${ }^{16}$

All the tested compounds (Table 4) showed good- to moderate anti-resorptive properties compared to the available BP-drug risedronate. In general, these data indicated only marginal differences for the potency of the tested BP products, due to the different arylidene substituents used. However, screening results showed that the most active BPs were found in the compounds $\mathbf{6 d}, \mathbf{j}$ that involve the dimethylamino-arylidine substituents, whereas the least activity appeared with compounds $\mathbf{6 e}, \mathbf{f}$ that contain the chloro-arylidine substituents.

In summary, the inhibition of bone resorption of the new synthesized BPs is significantly high when compared to the available BP-drug, risedronate (standard). Thus, the results obtained are encouraging for further optimization of the anti-resorptive properties of these compounds, and could be used to treat man in the future.

Table 4. Anti-resorptive Activity (ARA), Effective Dose $\left(E_{50}\right)$ of the BPs 6a-j and BP- acid 7

\begin{tabular}{ccccc}
\hline Compound & $\mathrm{R}_{1}$ & $\mathrm{R}_{2}$ & $\mathrm{Y}$ & $\begin{array}{c}\text { TPTX*-VitD3 } \\
\mathrm{ED} 50[\mu \mathrm{g} / \mathrm{kg}]\end{array}$ \\
\hline Risedronate & - & - & - & $12^{* *}$ \\
$\mathbf{6 a}$ & $\mathrm{H}$ & $\mathrm{Et}$ & $\mathrm{OH}-p$ & 48 \\
$\mathbf{6 b}$ & $\mathrm{H}$ & $\mathrm{Et}$ & $\mathrm{OH}-o$ & 38 \\
$\mathbf{6 c}$ & $\mathrm{H}$ & $\mathrm{Et}$ & $\mathrm{F}-p$ & 52 \\
$\mathbf{6 d}$ & $\mathrm{H}$ & $\mathrm{Et}$ & $\mathrm{N}(\mathrm{Me})_{2}-p$ & 22 \\
$\mathbf{6 e}$ & $\mathrm{H}$ & $\mathrm{Et}$ & $\mathrm{Cl}-o$ & 66 \\
$\mathbf{6 f}$ & $\mathrm{H}$ & $\mathrm{Et}$ & $\mathrm{Cl}-m$ & 64 \\
$\mathbf{6 g}$ & $\mathrm{Me}$ & $\mathrm{Et}$ & $\mathrm{OH}-p$ & 55 \\
$\mathbf{6 h}$ & $\mathrm{Me}$ & $\mathrm{Et}$ & $\mathrm{OH}-o$ & 50 \\
$\mathbf{6 i}$ & $\mathrm{Me}$ & $\mathrm{Et}$ & $\mathrm{F}-p$ & 28 \\
$\mathbf{6 j}$ & $\mathrm{Me}$ & $\mathrm{Et}$ & $\mathrm{N}(\mathrm{Me})_{2}-p$ & 16 \\
$\mathbf{7}$ & $\mathrm{Me}$ & $\mathrm{H}$ & $\mathrm{OH}-o$ & 33 \\
\hline
\end{tabular}

* All data are generated in the thyroparathyroidectomized (TPTX) acute in vivo rat model. ** This result was reproduced experimentally. 


\section{Conclusions}

The present investigations describe an efficient and simple approach to the synthesis of a variety of the title BPs in satisfactory yields with the use of easily available starting materials. The pharmacological evaluation discusses the anti-resorptive activities of the obtained products. Therefore, these compounds could be considered as lead molecules to be modified in order to improve the inhibition of bone resorption. Finally, although significant process has been made over the past decade, it is likely that realization of the full medical potential of both BPs and BPacids remains in the future, pending further advances in drug design.

\section{Experimental Section}

General Procedures. All melting points are uncorrected. IR spectra were recorded on a PerkinElmer spectrophotometer Model 297 using $\mathrm{KBr}$ discs. The ${ }^{1} \mathrm{H}$ - and ${ }^{13} \mathrm{C}-\mathrm{NMR}$ spectra were recorded on a JNM-GX-400 Fa Joel spectrometer, using TMS as internal reference. ${ }^{31} \mathrm{P}$ - NMR spectra were taken with a Varian CFT-20 (vs. external $85 \% \mathrm{H}_{3} \mathrm{PO}_{4}$ ). The mass spectra were obtainedd at $70 \mathrm{eV}$ on an MS-50 Kratos (A.E.I.) instrument provided with a data system. Elemental analyses were carried out at the Microanalysis Laboratory, Cairo University, Cairo, Egypt. Pharmacological evaluation was carried out in the Central Lab. Faculty of Agriculture, Banha University. The appropriate precautions in handing moisture-sensitive compounds were observed. Materials and reagents were purchased from Aldrich. The starting pyrimidines 4a-j were prepared as described in the literature. ${ }^{17}$

\section{Reactions of arylidenes 4a-j with tetra-ethyl methylene-1,1-bisphosphonate (1). Preparation of BPs 6a-j and 8a-f. General procedure}

To a stirred solution of $1.6 \mathrm{mmol}$ of 1 and $15 \mathrm{~mL} \mathrm{LiOH}(0.5 \mathrm{M})$ in $20 \mathrm{~mL}$ DMSO, was added slowly a solution of $1.1 \mathrm{mmol}$ of the substituted arylpyrimidines $4 \mathbf{4 a - j}$ in $15 \mathrm{~mL} D M S O$ at $0{ }^{\circ} \mathrm{C}$. The resulting mixture was stirred for $1 \mathrm{~h}$ and was then heated under reflux up to the consumption of the starting material for $\approx 2$ days (TLC). After removing of the solvent, $200 \mathrm{~mL}$ of distilled water and $\mathrm{HCl}(1 \mathrm{~N})$ was added $\left(\right.$ at $\left.-5^{\circ} \mathrm{C}\right)$ until the $\mathrm{pH}$ of the reaction mixture became acidic. The mixture was then extracted with AcOEt $(3 \times 50 \mathrm{~mL})$, and the combined organic phase was dried over anhydrous $\mathrm{MgSO}_{4}$. After removal of the solvent, under vacuum, the resulting residue was chromatographed on silica gel (n-hexane / ethyl acetate) to give BP-products 6a-j and 8a-f. Percentage yields; physical and spectral data of the products are listed in Tables 1-3.

Acid hydrolysis of BP (6h). Preparation of [2-(1,3-dimethyl-2,4,6-trioxohexahydropyrimidin-5-yl)-2-(2-hydroxyphenyl)ethane-1,1-diyl]-bis-(phosphonic acid) (7). The BP product $6 \mathbf{h}(0.5 \mathrm{~g})$ was dissolved in $20 \mathrm{~mL}$ conc. $\mathrm{HCl}$, and the mixture was then heated under reflux for $22 \mathrm{~h}$ (TLC). After cooling, the mixture was diluted, followed by evaporation to 
dryness under reduced pressure. After triturating with $\mathrm{CH}_{2} \mathrm{Cl}_{2}$, the solid was filtered and washed twice with ethanol / water (1:2, v/v) to give the analogue BP-acid (7). Percentage yield, physical and spectral data are listed in Tables 1-3.

Treatment of the reaction mixture $(1+4 a)$ with $p$-nitrobenzaldehyde. Preparation of diethyl [(1E)-3-(4-hydroxyphenyl)-1-(4-nitrophenyl)-3-(2,4,6-trioxo-hexahydropyrimidin-5-yl)prop-1-en-2-yl]phosphonate (10). To the reaction mixture (1+4a) in DMSO $(20 \mathrm{~mL})$ containing $\mathrm{LiOH}(15 \mathrm{~mL}, 0.5 \mathrm{M}), p$-nitrobenzaldehyde $(0.3 \mathrm{~g}, 2 \mathrm{mmol})$ was added. The reaction mixture was refluxed for $65 \mathrm{~h}$ (TLC). After the usual working up, the residual substance was extracted with AcOEt $(3 \times 50 \mathrm{~mL})$. The combined organic phase was dried over anhydrous $\mathrm{MgSO}_{4}$, followed by removal of the solvent, under vacuum. The resulting substance was recrystallized from EtOH to afford the monophosphonate product $\mathbf{1 0}$ as a sole product. Its yield, physical and spectral data are listed in Tables 1-3.

\section{Biological evaluation}

$\mathrm{ED}_{50}$ values ( $C F$. Table 4) for bone resorption biosynthesis inhibition in rats ('the rat model') were determined as described by Green et al. ${ }^{15}$

\section{Acknowledgements}

This research has been supported by the Egyptian Government (National Research Centre).

\section{References}

1. Fleisch, H. Breast Cancer Res. 2002, 4, 30.

2. Fleisch, H. Endocr. Rev. 1998, 19, 80.

3. Russell, R. G. G. Phosphorus, Sulfur, and Silicon 1999, 793, 144.

4. Shinoda, H.; Admerk, G.; Felix, R.; Fleisch, H.; Schenk, R.; Hagan, P. Calcif. Tissue Int. 1983, 35, 87 .

5. Schenk, R.; Merz, W. A.; Muhlbauer, R.; Russell, R. G. G.; Fleisch, H. Calc. Tissue Res. 1973, 11, 196.

6. Abdou, W. M.; Ganoub, N. A.; Elkhoshnieh, Y. O. Synlett 2003, 785.

7. Abdou, W. M.; Ganoub, N. A.; Geronikaki, A.; Sabry, E. Eur. J. Med. Chem. 2007, 675.

8. Abdou, W. M.; Khidre, M. D.; Sediek, A. A. Lett. Org. Chem. 2006, 3, 634.

9. Abdou, W. M.; Ganoub, N. A.; Fahmy, A. F.; Shaddy, A. A. Monatsh. Chem. 2006, 137, 105.

10. Silverstein, R. M.; Bassler, G. C.; Morrill T. C. Spectroscopic Identification of Organic Compounds, $4^{\text {th }}$ edn., John Wiley and Sons, Inc. New York, 1981. 
11. (a) Dufau, C.; Sturtz, G. Phosphorus, Sulfur, Silicon and Related Elements 1992, 69, 93; (b) Yuan, C.; Li, C. Phosphorus, Sulfur, and Silicon 1992, 69, 75.

12. Hutchinson, D. W.; Thornton, D. M. J. Organomet. Chem. 1988, 346, 341.

13. Sturtz, G.; Guervenou, J. Synthesis 1991, 661.

14. (a) Lehnert, W. Tetrahedron 1974, 30, 301. (b) Degenhard, C. R.; Burdsall, D. C. J. Org. Chem. 1986, 51, 3488 .

15. Green, J. R.; Müller, K.; Jaeggi, K. A. J. Bone Miner. Res. 1994, 9, 745.

16. (a) Fleisch, H. in Handbook of Experimental Pharmacology; Baker P.F. Ed;. Springer: Berlin/Heidelberg 1988, 83, pp 441. (b) Ebetino, F. H. M.; Francis, D.; Rogers, M. J.; Russell, R. G. G. Revs in Contemp. Pharmacother. 1998, 9, 233.

17. (a) Akabori, S. Chem. Ber. 1933, 66, 139. (b) Kaupp, G.; Jamal, R. N.; Schmeyers, J. Tetrahedron 2003, 59, 3753. (c) Xu, Y.; Dolbier, W. R. Tetrahedron 1988, 54, 6319. 